\title{
Data Acquisition System for ITER Neutron Diagnostic Divertor Neutron Flux Monitor
}

\author{
V. A. Fedorov, E. S. Martazov, Yu. A. Parishkin, N. A. Selyaev, ${ }^{1}$ Yu. A. Kashchuk and \\ ${ }^{1} \mathrm{~V}$. A. Vorobiev \\ National Research Nuclear University MEPhI, Institution "Project Center ITER"
}

The ITER nuclear diagnostics Divertor Neutron Flux Monitor (DNFM) is designed to determine the ITER fusion power. DNFM diagnostic provides a neutron yield and fusion power measurements for both deuterium-deuterium and deuterium-tritium plasmas with the required time resolution and uncertainty.

The data acquisition system (DAQ) is a part of the DNFM diagnostic. This system is designed for signal processing of the three neutron detectors modules, each including six fission chambers (FC).

The DAQ system performs the following functions:

- FCs bias generation and signals detection;

- transformation of the measurement data into neutron flux values at the location of the detector module;

- detectors module FCs and measurement channels calibration with external neutron source;

- data transfer to the upper systems - CODAC;

- diagnostics of measurement channels and their components, including a detector modules and a communication lines.

The structure of DNFM diagnostics in ITER is presented in Figure 1. There are three distributed subsystems runs under the CODAC. The green shows the DNFM diagnostic components and the blue shows the CODAC components.

The DNFM diagnostic used three detector modules located around the ITER Vacuum Vessel through 120 degrees. Detector modules are mounted on the Divertor Cassettes under the Dome. Each DNFM detector module consists of two detector units (DU) and each detector unit consists of three fission chambers. The first DU contains the FCs with a radiator of ${ }^{235} \mathrm{U}$ and the second DU contains the FCs with a radiator of high purity ${ }^{238} \mathrm{U}$. Fission chambers are manufactured with different sensitivities to satisfy ITER's requirements for time resolution, dynamic range and measurement uncertainty.

The DNFM Data Acquisition System consists of the three parallel independent subsystems (LPxx Subsystem) for the detector modules. Each Subsystem consists of the DAQ DNFM Control System Cubicle (CSC) and the DAQ DNFM Signal Conditioning Cubicle (SCC). The DAQ DNFM System Control Cubicle will be located in the Diagnostic Building and the DAQ DNFM Signal Conditioning Cubicle will be located in the Lower Ports (LP) in Tokamak Building.

The DNFM Data Acquisition System calculates the neutron flux at the location of the detector modules and transmits diagnostic measurements to the CODAC System every $1 \mathrm{~ms}$.

Data exchange with the CODAC system is performed on four networks: PON, SDN, DAN and TCN. There are two terminals linked to the CODAC system - the physicist terminal and the engineer terminal.

The DNFM diagnostic will be calibrated in situ using neutron sources during the brake in work of the reactor before the start of the nuclear phase (work with deuterium plasma). The expected dynamic range of neutron flux measurements by the divertor monitor in the operating mode is 7 orders of magnitude.

The DNFM data acquisition system is designed to work with an FC with a nominal bias voltage of up to $300 \mathrm{~V}$, an average pulse charge of about $2 \times 10^{-13} \mathrm{C}$ and a maximum output 
current of less than $3 \mathrm{~mA}$. Each FC is connected to the system in a differential way using triaxial cables with mineral insulation (MI cables) with an impedance of $50 \mathrm{Ohm}$ and a length of up to $20 \mathrm{~m}$. The FCs bias voltage settings are performed remotely. Each FCs power supply is independent in terms of control and monitoring and has remote access.

The measuring channels in the Signal Conditioning Cubicle are galvanically isolated from each other and from the power supply for a voltage of at least $1 \mathrm{kV}$.

To ensure the neutron flux dynamic range of 7 orders of magnitude the fission chambers signals processing concept used in this system presupposes the presence in one control channel of three processing methods - pulse, Campbelling (fluctuation) and current.

The neutron flux measurement period in the operating mode is less than $1 \mathrm{~ms}$. The settling time for the half-height level at the output of the measuring channel with a step change of the input signal in the measurement range does not exceed $1 \mathrm{~ms}$.

CSC consists of a PXI system, a data acquisition system Fast Controller (industrial PC), parts of the rack status monitoring kits and linkage with the CODAC System (Hab\&Cubicle health) and a switching component.

The Signal Processing Unit (SPU), components of switching and shielding of SPU from external influencing factors are placed in the SCC. The SPU consists of a pre-amplifier module, a control and preliminary processing module and a remote galvanic isolation module. The design provides for the possibility of removing the pre-amplifiers modules from the control and preliminary processing module for a distance of up to 30 meters. This will significantly reduce the amount of electronic equipment means to be shielded from hard radiation and exclude elements with high sensitivity to high-intensity magnetic fields.

In the SCC the pulses of the FCs are converted into voltage pulses, pre-processed and filtered. The analog signals of the measuring chains are converted to digital and transmitted to the CSC via fiber-optic communication lines.

In the CSC the data from the measurement chains are processed by processing and registration device. The processing and registration device consists of the PXI system based on NI PXIe-1075 and the Fast Controller and performs the following functions:

- control signals generation for the block of preliminary amplifiers;

- data collection from the normalizing converters of the measuring chains and their transformation into signals proportional to the neutron flux at the location of the detector module;

- calculation of the neutron flux at the location of the detector module in the entire of neutron flux measurement range;

- diagnostics of DAQ hardware;

- calibration of measuring channels.

The reduced count rate calculation of neutron pulses is performed for each chain and for the FC as a whole, taking into account the weight coefficients of the chains. The reduced count rate and neutron flux at the location of the detector module are also calculated. All calculated and measured data are transmitted to the CODAC system.

As a part of the development on the "Divertor Neutron Flux Monitor" diagnostic a mockup of the registration system for the DNFM detector module was produced. This mockup passed a number of laboratory and reactor tests which showed the efficiency of the presented solutions. So the non-linearity of the pulse chain was less than $1 \%$, and the non-linearity of the current and Campbell chains did not exceed $2 \%$. The tests also showed that the required performance in $1 \mathrm{~ms}$ with the current chain can be ensured only at currents of more than $100 \mathrm{nA}$.

Based on the mockup of the registration system for the DNFM detector module a threechannel system was developed and manufactured for the acceptance testing of the detector units.

Some of the solutions in part of CSC described above were adopted as basic when a use case example for ITER neutron diagnostics has been developed by ITER Organization. 